\title{
7 Literatur
}

In der nachstehenden Literaturliste sind einige Bücher und Artikel aufgeführt, mit denen der interessierte Leser (der nun hoffentlich kein Anfänger mehr in der objektorientierten Programmierung ist) seine Kenntnisse über objektorientierte Programmierung und Smalltalk weiter vertiefen kann.

Booch G (1994) Objet-oriented analysis and design with applications. 2nd edition. The Benjamin/Cummings Publishing Company, Inc., Redwood City, California

Gamma E, Helm R, Johnson R, Vlissides J (1995) Design Patterns: elements of reusable object-oriented software. Addison-Wesley, Reading, Massachusetts

Goldberg A (1984) Smalltalk-80. The Interactive Programming Environment. AddisonWesley, Reading, Massachusetts

Goldberg A und Robson D (1989) Smalltalk-80. The Language, Addison-Wesley, Reading, Massachusetts

Heeg G und .Bücker M (1994) Object Behavior Analysis - Von der Suche nach Objekten, OBJEKTspektrum, Vol. 2, 32-34, SIGS Publications, München

Lalonde WR, Pugh JR (1990) Inside Smalltalk. 2 Bd. Prentice Hall, Englewood Cliffs, New Jersey

Meyer B (1988) Object-Oriented Software Construction. Prentice Hall, Englewood Cliffs, New Jersey

Rubin K, Goldberg A (1992) Object Behavior Analysis. Communications of the ACM, Vol. 35(9)

Rumbaugh J, Blaha M, Premerlani W, Eddy F, Lorensen W (1993) Objektorientiertes Modellieren und Entwerfen. Carl Hanser Verlag, München, Wien, Prentice-Hall International Inc., London

Weitere Informationen über das Lagerhaltungsmodell aus Abschnitt 3.5 und viele andere Methoden zur Lösung von Optimierungsproblemen findet man in folgendem Einführungsbuch:

Neumann K, Morlock M (1992) Operations Research, Carl Hanser Verlag, München

Auf elektronischem Wege kann man sich z.B. unter den folgenden Adressen Ideen und Anregungen zu Smalltalk beschaffen:

st.cs.uiuc.edu oder mushroom.cs.man.ac.uk

(Ein Archiv mit Public-Domain Smalltalk-Quellcodes, das über ftp erreichbar ist.)

http://st-www.cs.uiuc.edu/

(Ein World Wide Web-Server.)

http://parcbench.parcplace.com/

(Ein World Wide Web-Server direkt von ParcPlace.) 